\title{
ISOLATION, CHARACTERIZATION AND ANTIBACTERIAL SCREENING OF ANTIBIOTICS PRODUCED FROM STREPTOMYCES ISOLATED FROM DUMPSITE SOILS IN ILORIN, NORTH CENTRAL NIGERIA
}

\author{
Risikat N. Ahmed ${ }^{1 a^{*}}$, Mercy O. Bamigboye ${ }^{2 b}$, Kamoldeen A. Ajijolakewu ${ }^{3 a}$, Sheriffdeen $O$. \\ Idris $^{4 a}$ and Nimat T. Ajide-Bamigboye ${ }^{5 a}$
}

\begin{abstract}
a Department of Microbiology, Faculty of Life Sciences, University of Ilorin, Kwara State, NIGERIA. Email: anrisikat@unilorin.edu.ng ${ }^{1}$; ajijolakewu382@gmail.com ${ }^{3}$; olajnr9@gmail.com ${ }^{4}$; nimattoyosi@yahoo.com ${ }^{5}$ bepartment of Industrial Chemistry, Faculty of Physical Sciences, University of Ilorin, Kwara State, NIGERIA. Email: obaleye.mo@unilorin.edu.ng ${ }^{2}$ *Corresponding Author: Risikat N. Ahmed Received: $11^{\text {th }}$ May $2020 \quad$ Accepted: $11^{\text {th }}$ Sep 2020 DOI: https://doi.org/10.22452/mjs.vol40no3.1

Published: $31^{\text {st }}$ Oct 2021
\end{abstract}

\begin{abstract}
Antibiotics are one of the most exploited metabolites produced by soil actinomycetes. This study isolated fifteen actinomycetes (A1 - A15) from dumpsite soils within Ilorin metropolis \& screened them for antibacterial activity. Isolates were identified with morphology \& biochemical characteristics. Their activity against clinical bacteria (Escherichia coli, Pseudomonas aeruginosa, Klebsiella pneumonia \& Staphylococcus aureus) was determined. Their bioactive metabolites after fermentation was assayed for by agar well diffusion \&compared with reference antibiotics. Molecular analysis of two most active isolates was done. The most active metabolite was subjected to GCMS. All isolates were identified as Streptomyces, 7 isolates exhibited activity against 3 selected human pathogen bacteria with highest $(10.5 \mathrm{~mm} \pm 0.35)$ by Streptomyces griseoplanus while $S$. aureus demonstrated total resistance to all isolates. Secondary activity revealed 7 of the metabolites as effective against the bacteria with highest activity $(28.5 \pm 1.04 \mathrm{~mm})$ by $S$. sparsogenes showing similar activity with Cefuroxime $(28.01 \pm 0.01 \mathrm{~mm})$. The two most active isolates were Streptomyces griseoplanus strain NR725RL-ISP 5009 \& Streptomyces sparsogenes strain NBRC 1308616S\& RNA nucleotide similarity revealed close phylogenic relationship. GCMS revealed10 compounds in S. sparsogenes strain NBRC 1308616S metabolite. Streptomyces from dumpsite soils within Ilorin produced bioactive compounds against clinical human pathogen bacteria and thus could be potential sources of antibiotics.
\end{abstract}

Keywords: S. sparsogenes strain NBRC 1308616S, Streptomyces griseoplanus strain NR725RLISP 5009, Antibiotics, Dumpsite soil, Antibacterial activity, Agar diffusion. 


\section{INTRODUCTION}

In the $20^{\text {th }}$ century, the idea of growth inhibition of one microorganism by another present in the same vicinity became mainstream. It was later discovered that such growth inhibition was mediated by secretion

of toxic metabolites later termed antibiotics (Singh et al., 2012). Over time, researchers have sought antibiotics from microorganisms isolated from several sources such as plant, soil, marine and freshwater environments. Among these, the soil ecosystem has been a major source of potential antibioticproducing microorganisms (Simon \& Daniel, 2011).

Bacteria genera, Streptomyces, Bacillus along with the fungal genera Penicillium and Cephalosporium are commonly found in soil. However, the genus Streptomyces is the most prolific antibiotic producers and they are a unique subgroup of bacteria called the actinomycetes (Ohnishi et al., 2008). Actinomycetes are gram positive bacteria consisting of a group of branching unicellular microorganisms of which Streptomyces are the most dominant.

Actinomycetes have over the years showed ability to produce a variety of bioactive secondary metabolites and for this reason; the discovery of novel antibiotic from them is becoming increasingly important (Dinos, 2017).
Of all bioactive secondary metabolites produced by microorganisms, $45 \%$ have been reported to be produced from actinomycetes, thus becoming a significant antibioticproducing genus exploited by pharmaceutical industries (Sharma et al., 2014). Industrially they have gained importance as antibiotics, anti-parasitic, antifungal, herbicides, pesticides, anticancer or immunosuppressive agents as well as industrially important enzymes (Sikander et al., 2018).

However, in the $21^{\text {st }}$ century, the problem of antibiotic resistance has become one of the leading threats to human existence. Thus, there is an urgent need to discover newer classes of antimicrobial agents effective against resistant pathogenic bacteria and fungi with minimum or no toxicity.

This study was conducted with the aim of isolating actinomycetes from dumpsite soils within Ilorin, north-central, Nigeria and screening them and their bioactive metabolites for antibacterial activity.

\section{MATERIALS AND METHODS}

\subsection{Sample Collection}

A total of 9 waste dumpsite soil samples were randomly collected from three different sites in Ilorin metropolis, North Central Nigeria. Samples were taken at a depth of $10 \mathrm{~cm}$, after clearing away $3 \mathrm{~cm}$ of top-soil (Aliero et al., 2017). These samples were air dried for 7 days at room temperature (Njenga et al., 2017). 


\subsection{Selective Isolation of Actinomycetes}

Isolation of actinomycetes was carried out as described by Bizuye et al. (2017) and Chaudhary et al. (2018) using starch casein agar (SCA) supplemented with Rifampicin $(2.5 \mu \mathrm{g} / \mathrm{ml})$ and nystatin $(50 \mu \mathrm{g} / \mathrm{ml})$. One gram of dried soil was suspended in $9 \mathrm{ml}$ sterile water and serially diluted up to $10^{-7}$. An aliquot of $0.1 \mathrm{ml}$ of each dilution was spread evenly over the surface of the prepared agar and incubated at $30^{\circ} \mathrm{C}$ for 7 days. Purified actinomycetes isolates were preserved on starch casein agar slant and incubated at $28^{\circ} \mathrm{C}$ for growth and preserved at $-4^{\circ} \mathrm{C}$ (Reddy et al., 2011).

\subsection{Identification of Actinomycetes}

\subsubsection{Morphological and Cellular Characterization}

The colonies of pure actinomycetes isolates were observed based on; color, aerial and substrate mycelia, surface texture and pigment production as described by Fawole and Oso (2007). The cellular characteristics were studied after Gram's staining techniques and viewing under a light microscope.

\subsubsection{Biochemical Characterization}

Colonies of pure actinomycetes were selected for biochemical tests such as; indole, catalase, methyl red, citrate utilization, starch hydrolysis, Voges-Proskaeur tests as described by Fay and Ba/rry (1974); Fawole \& Oso (2007); Abdulkadir \&Waliyu (2012).

\subsection{Human Pathogen Bacteria}

The human pathogen bacteria strains were obtained from the Teaching Hospital, University of Ilorin, Nigeria. They were; Klebsiella pneumoniae, Escherichia coli, Pseudomonas aeruginosa and Staphylococcus aureus. These were streaked on nutrient agar plates and incubated at $37^{\circ} \mathrm{C}$ for 24 hours to obtain fresh colonies and subsequently maintained on nutrient agar slants at $4^{\circ} \mathrm{C}$.

\subsection{Primary Antibacterial Screening}

Primary screening was performed using cross streak method as described by Bizuye et al. (2013). Metabolite-producing isolates were inoculated as vertical straight lines on Mueller Hinton agar (MHA) and incubated at $28^{\circ} \mathrm{C}$ for 5-7 days after which human pathogen bacteria were inoculated perpendicularly and incubated at $37^{\circ} \mathrm{C}$ for 24 hours. Zones of inhibition were measured and recorded.

\subsection{Secondary Antibacterial Screening \\ 2.6.1 Fermentation and Extraction of Bioactive metabolite from Streptomyces}

Bioactive compounds were produced using actinomycetes isolates in a submerged fermenter. They were separately grown in $100 \mathrm{~mL}$ starch casein broth and incubated at $30^{\circ} \mathrm{C}$ for 7 days. Following incubation, $10 \%$ of the broth culture was inoculated in an 
antibiotic production medium consisting: soluble starch $25 \mathrm{~g}$, glucose $10 \mathrm{~g}$, yeast extract $2 \mathrm{~g}, \mathrm{CaCO}_{3} 3 \mathrm{~g}$, Trace salts solution $1 \mathrm{ml}, \mathrm{pH}$ $7.5 \pm 0.2$ (Trace salt solution- $\mathrm{FeSO}_{4} .7 \mathrm{H}_{2} \mathrm{O}-$ $0.5 \mathrm{~g}, \mathrm{CuSO}_{4} .5 \mathrm{H}_{2} \mathrm{O}-0.5 \mathrm{~g}, \mathrm{ZnSO}_{4} .7 \mathrm{H}_{2} \mathrm{O}-0.5$ $\mathrm{g}, \mathrm{MnCl}_{2} .4 \mathrm{H}_{2} \mathrm{O}-0.5 \mathrm{~g}$ in $100 \mathrm{ml}$ of distilled water). The flasks were shaken at $28^{\circ} \mathrm{C}$ and $160 \mathrm{rpm}$ for 5-7 days on a rotary shaker after fermentation the broth was centrifuged at 4000rpm for 20 minutes to remove cells and obtain a clear supernatant containing the metabolite (Ashok \& Karpagam, 2016). This was mixed with equal volume of $95 \%$ ethanol on a rotatory shaker for $24 \mathrm{~h}$. The solvent phase was evaporated in water bath at $40^{\circ} \mathrm{C}$ to obtain dried partially purified bioactive metabolite which was redissolved in DMSO to obtain a concentration of $100 \mu \mathrm{l}$ and stored at $4^{\circ} \mathrm{C}$ for further studies (Raja \& Prabakarana, 2011; Chaudhary et al., 2018).

\subsubsection{Determination of Antibacterial Activity}

Antibacterial activity of bioactive metabolite was determined by agar well diffusion method (Ahmed et al., 2015). Cell concentration of all test microorganisms were adjusted to $0.5 \mathrm{McF}$ arland turbidity standards. Muller Hinton agar plates were prepared and spread with some human pathogen bacteria using a sterile cotton swab. Wells were prepared using sterile cork borer. A volume of $100 \mu \mathrm{l}$ of the bioactive metabolite was dispensed into each well and allowed to diffuse for 2 hours and incubated at $37^{\circ} \mathrm{C}$ for 24 hours. Following incubation, zone of inhibition around each well was recorded (Kaur et al., 2016) and a comparative study of antibiotic susceptibility was done using Multi-disc (Oxoid) containing standard antibiotics against human pathogen bacteria selected.

\subsection{Molecular Identification of Most Potent Actinomycetes}

The identities of the two most active isolates were confirmed by the sequence analysis of the small subunit rRNA genes. Following manufacturer's guide, the QIAamp DNA Mini Kit (250) (cat No. 51306) was used for the isolation of the genomic DNA. Sequences of the small subunit $16 \mathrm{~S}$ rRNA genes were amplified using the standard sets of primers 16SF (GTGCCAGCAGCCGCGCTAA) and 16SR (AGACCCGGGAACGTATTCAC) to obtain an amplicon size of $1500 \mathrm{bp}$. Amplification was carried out in a $25 \mu 1$ total reaction mixture consisting of 10x PCR buffer, $2.5 \mu 1$; 2.0 $\mu 1 ; 2.5$ MmDNTPs, $0.1 \mu \mathrm{l} ; 5.0 \mathrm{u} / \mu \mathrm{lTaq}$ DNA polymerase, $0.2 \mu \mathrm{l} ; 10 \mathrm{ng} / \mu \mathrm{l}$ of each primer, 3.0 $\mu \mathrm{l}$; template DNA, $1.0 \mu \mathrm{l}$ and deionized distilled water $13.4 \mu \mathrm{l}$ in a BioRad (USA) thermal cycler at $94^{\circ} \mathrm{C}$ for 5 minutes (Initial denaturation), $94^{\circ} \mathrm{C}$ for $30 \mathrm{sec}$ (denaturation), $72^{\circ} \mathrm{C}$ for $45 \mathrm{sec}$ (extension) and $72^{\circ} \mathrm{C}$ for 7 minutes (final extension). The total number of cycles was 36 , with the final extension of $72^{\circ} \mathrm{C}$ for 7 minutes. To ascertain successful amplification, PCR products (50 $\mu \mathrm{l})$ were size-separated by electrophoresis on a $1 \%$ agarose gel prepared in $1 \%$ TAE buffer 
containing $0.5 \mu \mathrm{l} / \mathrm{ml}$ ethidium bromide and visualized under transilluminator (BioRad, USA) against 1000bp DNA ladder (Genei). The PCR product (1500bp) was purified from contaminating products by electro-elution of the gel slice containing the excised desired fragments with Qiaquick gel extraction kit (Qiagen, USA) in a $300 \mu \mathrm{l}$ of nuclease free water. Product was diluted in Tris buffer (10 $\mathrm{mM}, \mathrm{pH} 8.5$ ) at $1: 1000$ to $30 \mathrm{ng} / \mu \mathrm{l}$ prior to Sanger sequencing using automated sequencer (ABI PRISM 310, Applied Biosystems, USA). Sequences of each isolates were subjected to similarity check with previously identified species by using BLASTn tool on the NCBI database (www.ncbi.nlm.nih.gov:80/BLASTN/) to identify the isolates up to strain level.

\subsection{Gas Chromatography - Mass Spectrometry (GC-MS)}

Identification of compounds present in the metabolite using GC-MS were based according to the molecular mass, peak observed, and molecular chemical formula which are directly proportional to the amount of molecules present in the band. Only the most effective metabolite (A10) produced by S. sparsogens was further analyzed by GCMS (GC-MS TQ 8050; Schimadzu, Johannesburg, South Africa) as described by Mangayi et al (2019). The metabolite was passed into the Mass Spectroscopy through Gas Chromatography, and ionized into the gaseous ions state in the ionization source.
This was thereafter passed into the mass analyzer. The ions observed with different mass per charge ratios were separated out into the electron multiplier by indicating an electrical signal to determine the identity of compounds (Narendhran et al., 2014). The identities of compounds were determined by searching known molecules in data bases.

\subsection{Statistical Analysis of Data}

Statistical significance was determined using ordinary one-way analysis of variance (ANOVA), while multiple comparisons between means were determined by Tukey's multiple comparisons test. Analysis was performed using Sigma Plot for Windows version 10.0 (SysStat Softwares Inc.). All data were expressed as means of triplicates $\pm \mathrm{SE}$.

\section{RESULTS}

\subsection{Recovery and Identification of Streptomyces from Soil Samples}

A total of 15 Streptomyces species were isolated from nine (9) soil samples and coded A1 - A15. According to morphological characterization, they exhibited varying elevations, pigments, edge, textures, shape (Table 1). The biochemical characteristics revealed that all isolates were Streptomyces species and they were urease positive, indole negative while other biochemical parameters differed with species (Table 2). 
Table 1. Colony Morphology of Streptomyces Isolates

\begin{tabular}{|c|c|c|c|c|c|c|c|}
\hline ISOLATES & ELEVATION & PIGMENT & EDGE & TEXTURE & SURFACE & OPTICAL & SHAPE \\
\hline A1 & Convex & Whitish & Smooth & Powdery & Smooth & Transparent & Circular \\
\hline A2 & Flat & Pink & Smooth & $\begin{array}{l}\text { Sticky and } \\
\text { Powdery }\end{array}$ & Smooth & Opaque & Irregular \\
\hline A3 & Raised & $\begin{array}{l}\text { Blackish } \\
\text { green deep }\end{array}$ & Rough & Powdery & Smooth & Opaque & Irregular \\
\hline A4 & Convex & Green & Smooth & Powdery & Rough & Opaque & Irregular \\
\hline A5 & Raised & $\begin{array}{l}\text { Golden } \\
\text { yellow }\end{array}$ & Smooth & Powdery & Rough & Opaque & Circular \\
\hline A6 & Raised & $\begin{array}{l}\text { Yellowish } \\
\text { green }\end{array}$ & Smooth & Powdery & Rough & Transparent & Irregular \\
\hline A7 & Raised & $\begin{array}{l}\text { Greenish } \\
\text { white }\end{array}$ & Smooth & Sticky & Smooth & Opaque & Circular \\
\hline A8 & Flat & Orange & Undulating & Smooth & Smooth & Opaque & Round \\
\hline A9 & Raised & Cream & Entire & Smooth & Rough & Opaque & Round \\
\hline A10 & Raised & Green & Undulating & Rough & Rough & Opaque & Irregular \\
\hline A11 & Flat & White & Undulating & Mucoidal & Smooth & Opaque & Round \\
\hline A12 & Raised & Black & Rough & Powdery & Rough & Transparent & Rhizoid \\
\hline A13 & Convex & Brown & Smooth & Smooth & Smooth & Transparent & Irregular \\
\hline A14 & Flat & Grey & Rough & Rough & Smooth & Opaque & Irregular \\
\hline A15 & Raised & Creamy & Rough & Mucoidal & Smooth & Transparent & Irregular \\
\hline
\end{tabular}


Table 2. Identification of Streptomyces by Biochemical Characterization

\begin{tabular}{|c|c|c|c|c|c|c|c|c|c|c|c|}
\hline ISOLATES & $\begin{array}{l}\frac{1}{2} \\
\text { そ }\end{array}$ & $\frac{\pi}{\sqrt{2}}$ & 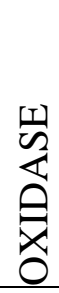 & 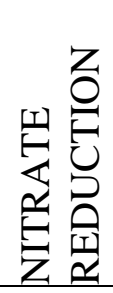 & 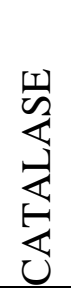 & 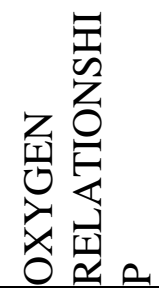 & 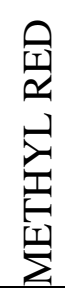 & 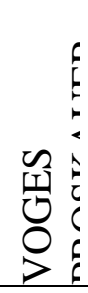 & 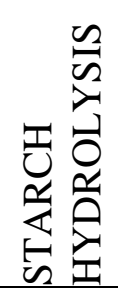 & 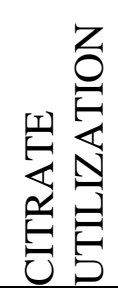 & TENTATIVE IDENTITY \\
\hline A1 & - & + & + & + & + & $\mathrm{A}$ & - & - & + & - & $\begin{array}{l}\text { Streptomyces } \\
\text { glaucescens }\end{array}$ \\
\hline $\mathrm{A} 2$ & - & + & + & + & + & $\mathrm{A}$ & - & - & + & - & Streptomyces sp. \\
\hline A3 & - & + & + & + & + & A & - & - & + & - & Streptomyces sp. \\
\hline A4 & - & + & + & + & + & A & - & - & + & - & Streptomyces sp. \\
\hline A5 & - & + & + & + & + & A & - & - & + & - & Streptomyces sp. \\
\hline A6 & - & + & + & + & - & A & - & - & + & - & $\begin{array}{l}\text { Streptomyces } \\
\text { griseoplanus }\end{array}$ \\
\hline A7 & - & + & + & + & + & A & - & - & + & - & $\begin{array}{l}\text { Streptomyces } \\
\text { griseoflavus }\end{array}$ \\
\hline A8 & - & + & + & + & + & A & - & - & + & + & Streptomyces cyaneus \\
\hline A9 & - & + & + & + & + & A & - & - & - & - & Streptomyces sp. \\
\hline A10 & - & + & + & + & + & A & - & - & + & - & $\begin{array}{l}\text { Streptomyces } \\
\text { sparsogenes }\end{array}$ \\
\hline A11 & - & + & + & + & + & A & - & - & - & - & $\begin{array}{l}\text { Streptomyces } \\
\text { diastaticus }\end{array}$ \\
\hline A12 & - & + & + & + & - & A & - & - & + & - & Arthrobacter \\
\hline A13 & - & + & + & + & + & A & + & + & + & - & $\begin{array}{l}\text { Streptomyces } \\
\text { atroolivaceus }\end{array}$ \\
\hline A14 & - & + & + & + & - & A & - & - & - & - & Steptomyces flaveolus \\
\hline A15 & - & + & + & + & + & A & + & - & + & - & Streptomyces sp. \\
\hline
\end{tabular}

Keys: +: Positive reaction; -: Negative reaction; A: Aerobic 


\subsection{Primary Screening of Streptomyces Isolated from Soil}

The result of primary screening of fifteen (15) Streptomyces species against four (4) human pathogen bacteria revealed that only seven (7) of these ( $S$. glaucescens, $S$. griseoplanus, S. griseoflavus, S. cyaneus, $S$. sparsogenes, $S$. diastaticus, and S. flaveolus) exhibited antibacterial activity against three of the human pathogenic bacteria (E. coli, $K$. pneumonia \& P. aeruginosa). All the seven active Streptomyces species exhibited inhibitory action against $P$. aeruginosa with the highest activity $(10.5 \mathrm{~mm} \pm 0.35)$ shown by Streptomyces griseoplanus while Staphylococcus aureus demonstrated total resistant to all seven active isolates without any zones of inhibition (Table 3).

Table 3. Primary Screening of Isolates against some Human Pathogen Bacteria

\begin{tabular}{|c|c|c|c|c|c|c|c|c|c|c|c|c|c|c|c|}
\hline \multicolumn{16}{|c|}{ Mean diameter zone of inhibition (mm) } \\
\hline $\begin{array}{l}\text { Test } \\
\text { Organ } \\
\text { ism }\end{array}$ & A1 & A2 & A3 & A4 & A5 & A6 & A7 & A8 & A9 & A10 & A11 & A12 & A13 & A14 & A15 \\
\hline E. coli & $\begin{array}{l}3.50 \pm \\
0.35\end{array}$ & $\begin{array}{l}0.00 \pm \\
0.00\end{array}$ & $\begin{array}{l}0.00 \pm \\
0.00\end{array}$ & $\begin{array}{l}0.00 \pm \\
0.00\end{array}$ & $\begin{array}{l}0.00 \pm \\
0.00\end{array}$ & $\begin{array}{l}2.50 \pm \\
0.35\end{array}$ & $\begin{array}{l}1.50 \pm \\
0.35\end{array}$ & $\begin{array}{l}5.50 \pm \\
0.35\end{array}$ & $\begin{array}{l}0.00 \pm \\
0.00\end{array}$ & $\begin{array}{l}5.50 \pm \\
0.35\end{array}$ & $\begin{array}{l}5.50 \pm \\
1.06\end{array}$ & $\begin{array}{l}0.00 \pm \\
0.00\end{array}$ & $\begin{array}{l}0.00 \pm \\
0.00\end{array}$ & $\begin{array}{l}0.00 \pm \\
0.00\end{array}$ & $\begin{array}{l}0.00 \pm \\
0.00\end{array}$ \\
\hline $\begin{array}{l}S . \\
\text { aureus }\end{array}$ & $\begin{array}{l}0.00 \pm \\
0.00\end{array}$ & $\begin{array}{l}0.00 \pm \\
0.00\end{array}$ & $\begin{array}{l}0.00 \pm \\
0.00\end{array}$ & $\begin{array}{l}0.00 \pm \\
0.00\end{array}$ & $\begin{array}{l}0.00 \pm \\
0.00\end{array}$ & $\begin{array}{l}0.00 \pm \\
0.00\end{array}$ & $\begin{array}{l}0.00 \pm \\
0.00\end{array}$ & $\begin{array}{l}0.00 \pm \\
0.00\end{array}$ & $\begin{array}{l}0.00 \pm \\
0.00\end{array}$ & $\begin{array}{l}0.00 \pm \\
0.00\end{array}$ & $\begin{array}{l}0.00 \pm \\
0.00\end{array}$ & $\begin{array}{l}0.00 \pm \\
0.00\end{array}$ & $\begin{array}{l}0.00 \pm \\
0.00\end{array}$ & $\begin{array}{l}0.00 \pm \\
0.00\end{array}$ & $\begin{array}{l}0.00 \pm \\
0.00\end{array}$ \\
\hline $\begin{array}{l}K . \\
\text { pneum } \\
\text { oniae }\end{array}$ & $\begin{array}{l}4.50 \pm \\
0.35\end{array}$ & $\begin{array}{l}0.00 \pm \\
0.00\end{array}$ & $\begin{array}{l}0.00 \pm \\
0.00\end{array}$ & $\begin{array}{l}0.00 \pm \\
0.00\end{array}$ & $\begin{array}{l}0.00 \pm \\
0.00\end{array}$ & $\begin{array}{l}8.50 \pm \\
0.35\end{array}$ & $\begin{array}{l}3.50 \pm \\
0.35\end{array}$ & $\begin{array}{l}9.50 \pm \\
0.35\end{array}$ & $\begin{array}{l}0.00 \pm \\
0.00\end{array}$ & $\begin{array}{l}6.00 \pm \\
0.7\end{array}$ & $\begin{array}{l}5.50 \pm \\
0.35\end{array}$ & $\begin{array}{l}0.00 \pm \\
0.00\end{array}$ & $\begin{array}{l}0.00 \pm \\
0.00\end{array}$ & $\begin{array}{l}0.00 \pm \\
0.00\end{array}$ & $\begin{array}{l}0.00 \pm \\
0.00\end{array}$ \\
\hline $\begin{array}{l}P . \\
\text { aerugi } \\
\text { nosa }\end{array}$ & $\begin{array}{l}7.50 \pm \\
0.35\end{array}$ & $\begin{array}{l}0.00 \pm \\
0.00\end{array}$ & $\begin{array}{l}0.00 \pm \\
0.00\end{array}$ & $\begin{array}{l}0.00 \pm \\
0.00\end{array}$ & $\begin{array}{l}0.00 \pm \\
0.00\end{array}$ & $\begin{array}{l}10.50 \\
\pm 0.35\end{array}$ & $\begin{array}{l}2.50 \pm \\
0.35\end{array}$ & $\begin{array}{l}6.50 \pm \\
0.35\end{array}$ & $\begin{array}{l}0.00 \pm \\
0.00\end{array}$ & $\begin{array}{l}6.50 \pm \\
0.35\end{array}$ & $\begin{array}{l}6.50 \pm \\
0.35\end{array}$ & $\begin{array}{l}0.00 \pm \\
0.00\end{array}$ & $\begin{array}{l}0.00 \pm \\
0.00\end{array}$ & $\begin{array}{l}3.50 \pm \\
0.35\end{array}$ & $\begin{array}{l}0.00 \pm \\
0.00\end{array}$ \\
\hline
\end{tabular}

Key: Values represented are means of triplicates \pm SEM 


\subsection{Secondary Screening of Active} Metabolites from Streptomyces against some human Pathogen Bacteria

The results obtained indicated that seven (7) metabolites produced from the active Streptomyces showed zones of inhibition against human pathogen bacteria with highest antibacterial activity $(28.5 \pm 3.54 \mathrm{~mm})$ by $S$. sparsogenes strain NBRC $1308616 S$ at a concentration of $100 \mu \mathrm{l}$ against Staphylococcus aureus (Figure 1) which was the only resistant bacterium in the primary assay. Sensitivity plates of inhibition zones of active metabolites against $P$. aeruginosa is shown below (Plate1).

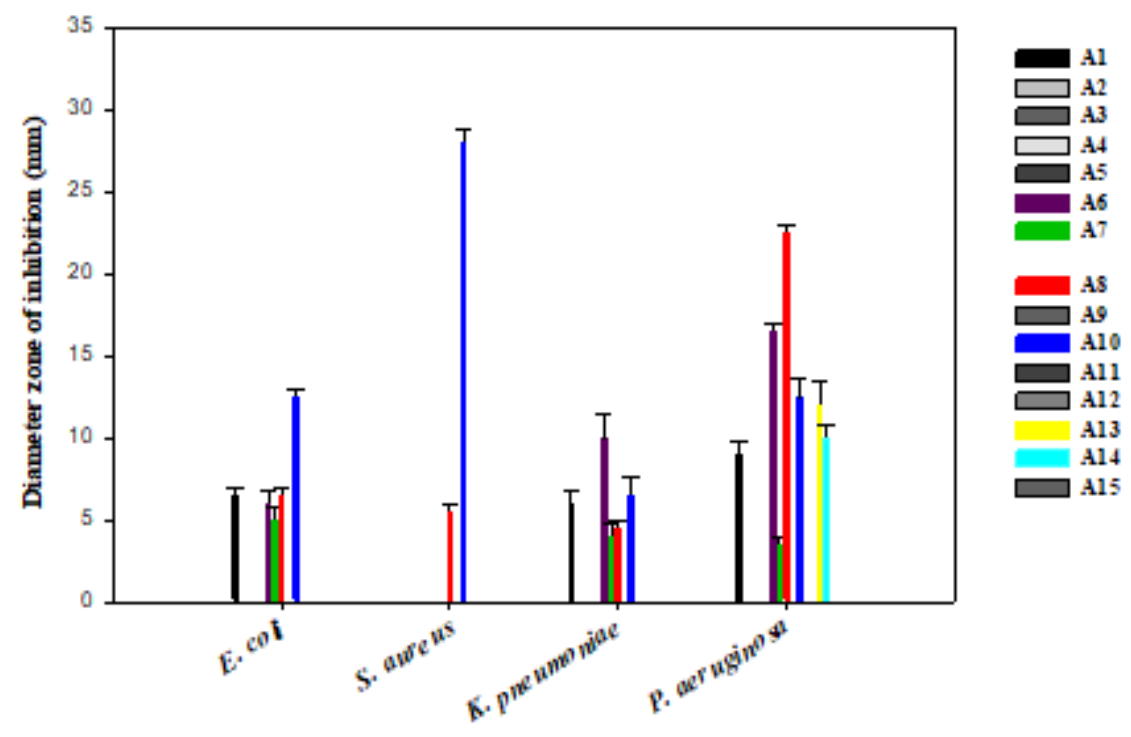

Figure 1. Secondary screening of metabolite from isolates against some selected human pathogen bacteria

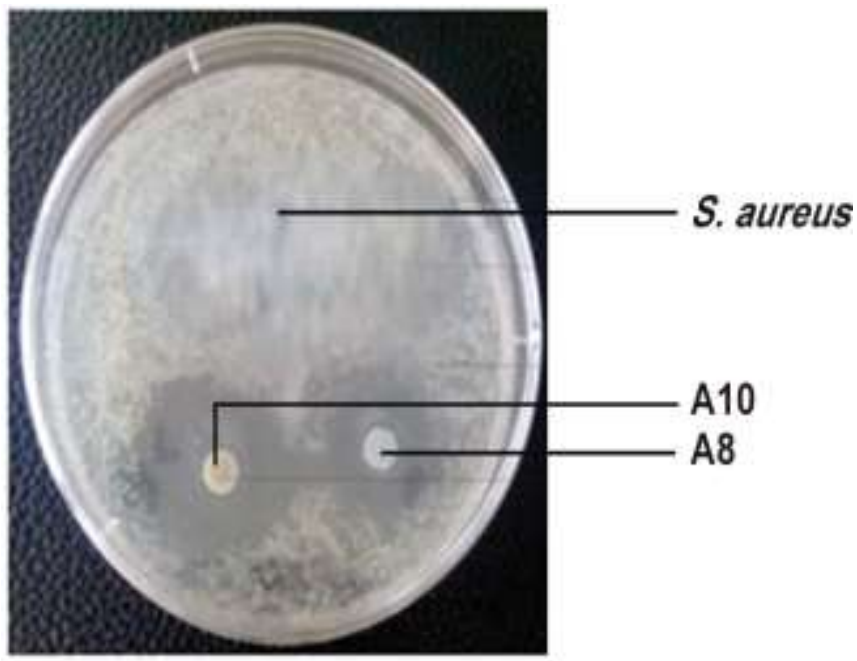

Plate 1. Sensitivity plate showing inhibition of $S$. aureus by metabolites from 2 Streptomyces spp. 


\subsection{Antibacterial Activity of Reference Antibiotics against selected Human Pathogen Bacteria}

Generally, the reference antibiotics showed inhibitory activities similar with those from active metabolites against human pathogen bacteria, however, not all the antibiotics exhibited inhibitory activity towards the human pathogen bacteria. ciprofloxacin and augmentin were totally resisted by all four human pathogen bacteria hence showing no zones of inhibition. The highest antibacterial activity $(28.01 \pm 0.01 \mathrm{~mm})$ was observed by Cefuroxime against Escherichia coli (Table 4).

Table 4. Antibacterial activity of reference antibiotics against human pathogen strains

\begin{tabular}{ccccc}
\hline Reference & \multicolumn{4}{c}{ Diameter of zone of inhibition $(\mathbf{m m})$} \\
antibiotic & SA & KP & PA & EC \\
\hline CAZ & $14.5 \pm 0.70$ & - & - & - \\
CRX & $27.5 \pm 4.95$ & $25.0 \pm 2.83$ & - & $28.01 \pm 0.01$ \\
GEN & $20.5 \pm 2.12$ & $19.0 \pm 0.00$ & - & $17.0 \pm 1.41$ \\
CTR & - & - & $27.0 \pm 2.83$ & - \\
ERY & $18.5 \pm 4.95$ & $18.5 \pm 3.54$ & - & $15.0 \pm 1.41$ \\
CXC & - & - & - & - \\
OFL & $21.5 \pm 0.71$ & $24.5 \pm 0.71$ & $25.5 \pm 2.12$ & $26.5 \pm 0.71$ \\
AUG & - & - & - & - \\
\hline
\end{tabular}

(-) sign indicates no zone formation (no antibiotic activity); all values are mean $\pm \mathrm{St}$ Dev

Key: $\mathbf{C A Z}=$ Ceftazidime, $\mathbf{E R Y}=$ Erythromycin $\mathbf{C R X}=$ Cefuroxime, $\mathbf{C X C}=$ Ciprofloxacin, $\mathbf{G E N}$ $=$ Gentamycin, $\mathbf{O F L}=$ Ofloxacin, $\mathbf{C T R}=$ Ceftriaxone, $\mathbf{A U G}=$ Augmentin $\mathbf{S A}=$ Staphylococcus aureus, $\mathbf{K P}=$ Klebsiella pneumoniae, $\mathbf{P A}=$ Pseudomonas aeruginosa, $\mathbf{E C}=$ Escherichia coli

3.5 Molecular Identification of two most active Streptomyces

The molecular characterization of the two most active Streptomyces species during primary and secondary screening assays (A6 and A10) revealed their identity as $S$.
Streptomyces griseoplanus strain NR725RLISP 5009 and $S$. sparsogenes strain NBRC 1308616S respectively. Their DNA extraction ladder as shown by gel electrophoresis is given in Plate 2 and the phylogenetic tree of their sequences showed that they are closely related (Figure 2).

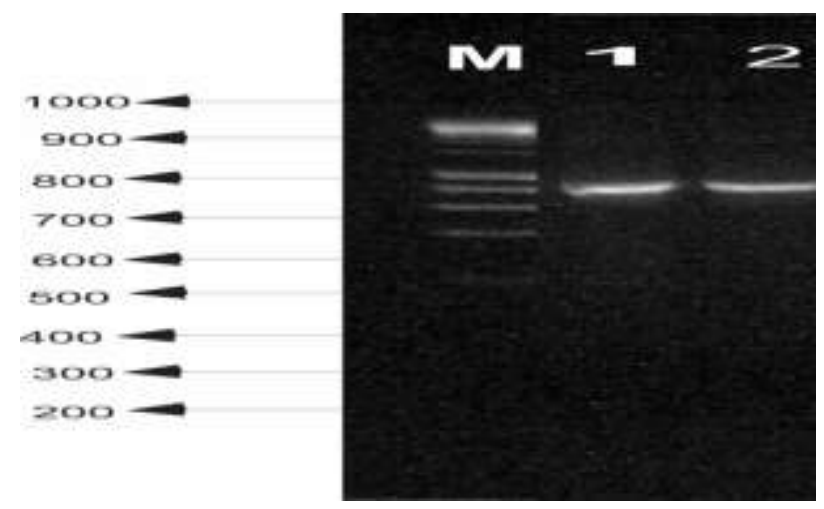

Plate 2. DNA extraction ladder of (1) Streptomyces griseoplanus strain NR725RL-ISP 5009(2) Streptomyces sparsogenes strain NBRC 1308616S with band size between 200-1000 kda 


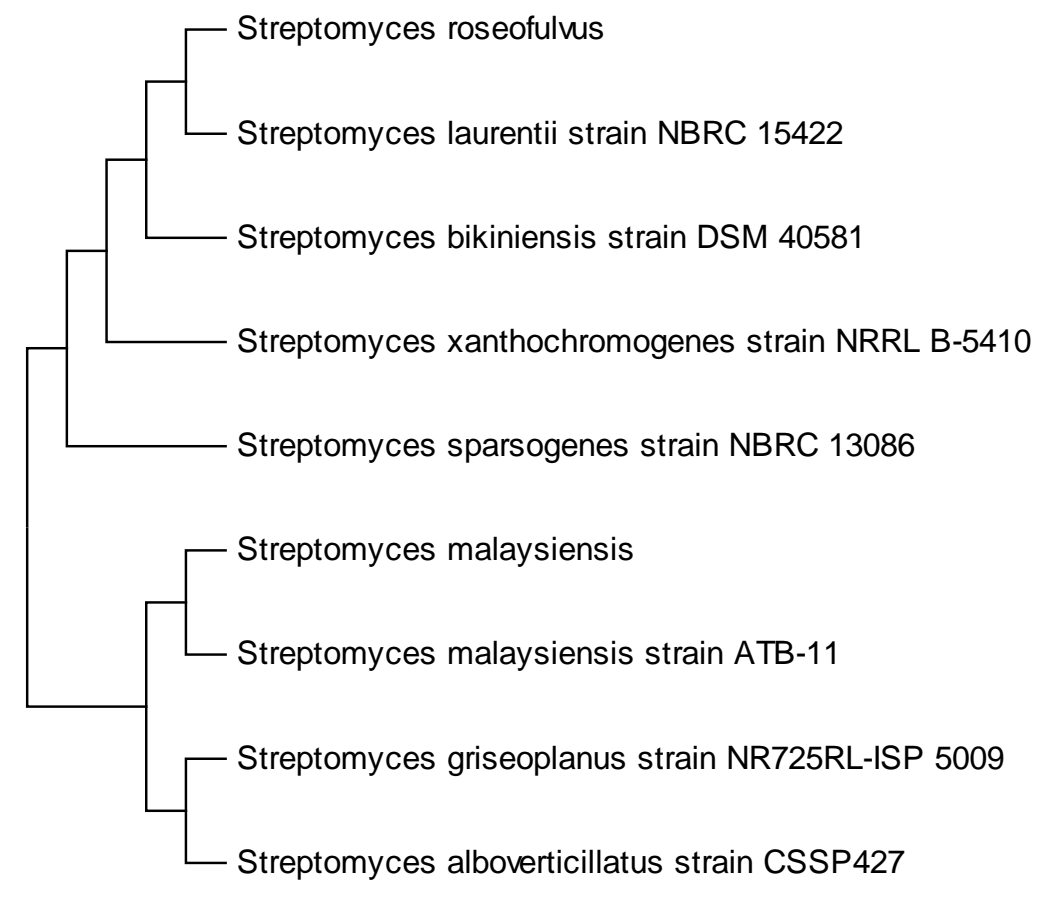

Figure 2. Molecular phylogenetic analysis using the maximum likelihood method

\subsection{Gas Chromatography - Mass Spectrometry (GC-MS)}

As previously stated, the most effective metabolite (A10) analyzed by GC-
MS revealed the presence of 10 compounds out of which fumaric acid was also detected followed by several peaks identified as a cyclic volatile organosilicone compounds (Table 3).

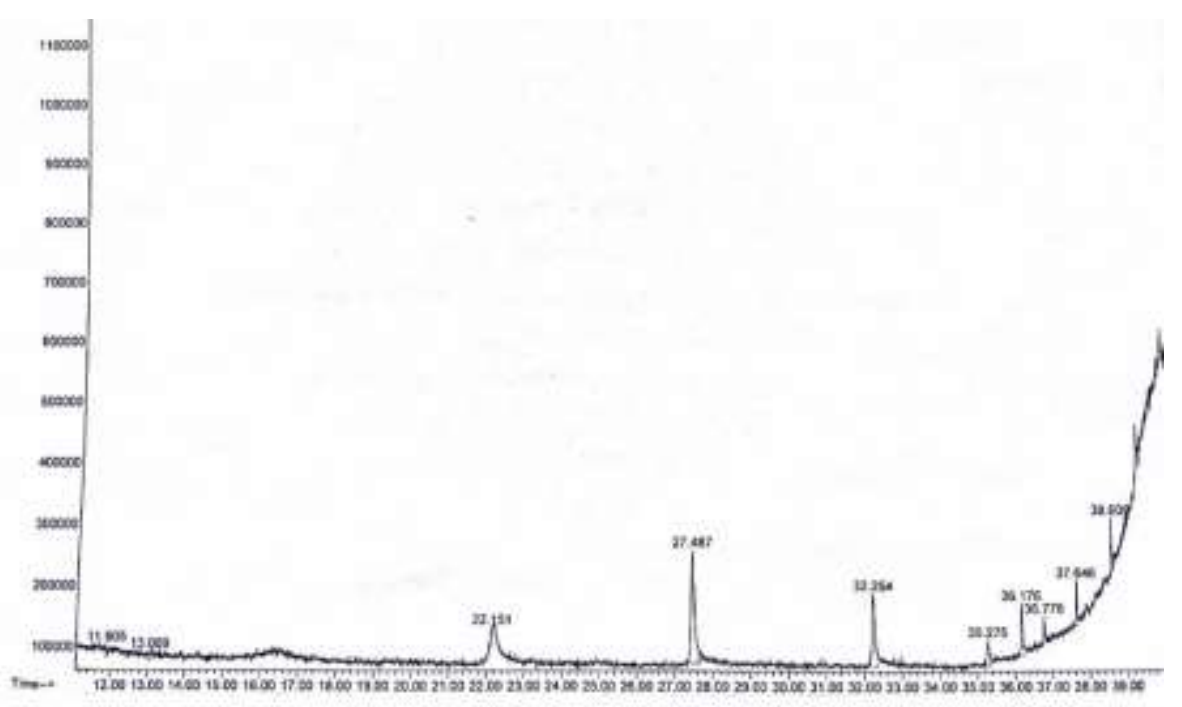

Figure 3. GC-MS spectra of bioactive metabolite from Streptomyces sparsogenes strain NBRC 1308616S 
Table 5. Name and Structure of Compounds from Bioactive metabolite of Streptomyces sparsogenes strain NBRC 1308616S Identified by GC-MS

S/N Name of the compound Structure

(5-Ethoxycarbonylamino-2,6-

1 dimethylpyridin-3-yl

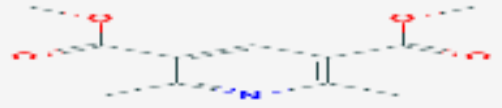

2 2-p-Tolyl-2,3-dihydro-1H-bezo(1,3,2)

diazaborole

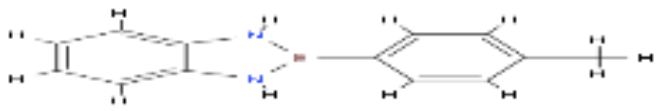

3 Cyclohexasiloxane, dodecamethyl

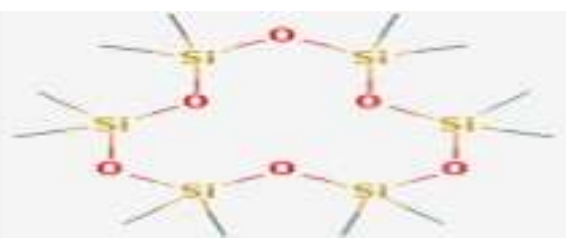

4 Cycloheptasiloxane, tetradecamethyl

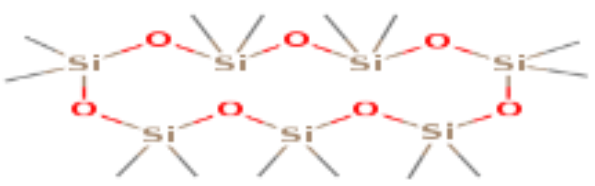

$5 \quad$ Cyclooctasiloxane, hexadecamethyl

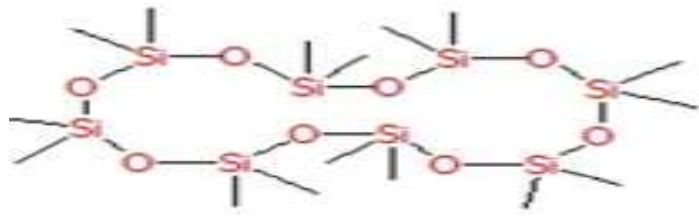

$6 \quad$ Fumaric acid, 3-hexyl undecyl este

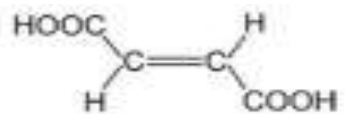

7 Cyclononasiloxane, octadecamethyl 

8 Silane, diethylpentadecyloxy(2-

9 Cyclodecasiloxane, eicosamethyl

10

Hexasiloxane, 1,1,3,3,5,5,7,7,9,9,11,11-
dodecamethyl

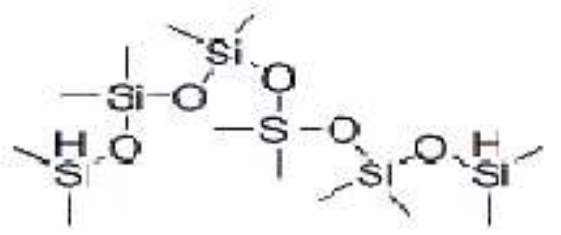

\section{DISCUSSION}

Actinomycetes from different sources have been identified as a major producer of a wide range of bioactive substances (Sharma et al., 2014), however, there have been very little or no report regarding screening of extracted bioactive compounds from them in Ilorin, north-central Nigeria.

This present study showed that Streptomyces species were isolated within Ilorin metropolis and their morphological and biochemical characteristics were in line with existing literatures hence confirming their identity. According to a research by Sathi et al. (2001) Streptomyces growth is characterized by small compact and soft colonies tightly attached to the surrounding agar.

The primary screening assay revealed that some of the isolates from this study exhibited antibacterial activity against some selected human pathogen bacteria used except for S. aureus while Secondary screening of the product of fermentation of the isolates yielded better activity than did the primary and as a result $S$. aureus which was resistant in the primary assay became susceptible in the secondary screening. Large scale production of antibiotics depends majorly on fermentation because during the process producer organisms multiply rapidly in a free flowing medium with optimized nutrient composition to support and enhance the secretion of copious quantities of the desired bioactive components relative to the quantity produced during primary screening. Further, it has been earlier argued that screening techniques based on the cultivation of live organisms on solid agar substrates is often affected by certain extraneous factors such as other cell lysates there by giving false positive results (Sharma et al., 1986). Hence, the increased concentrations of potent bioactive compounds during fermentation propels it as a reliable antibiotic screening technique for actinomycetes species which was also reflected when compared with the assay of standard reference antibiotics. This may have been responsible for the increased activity demonstrated by the best producer strain. Thus suggesting fermentation as a better 
means of obtaining quality antibacterial extract from microorganisms.

Molecular characterization serves as a veritable tool in the accurate identification of organisms and in this study it revealed the evolutionary relationship of the identified species showing them to be closely related on the phylogenetic tree. According to separate earlier reports by Ziemert \& Jensen (2012) and Ajijolakewu et al. (2016), the molecular phylogeny uses sequenced data to infer evolutionary relationships between organisms and the genes they maintain. In line with this suggestion, Ziemert \& Jensen (2012) had earlier opined that phylogenetic relationships among organisms provides unalloyed information which delineate the architecture and function of the genes involved in the biosynthesis of similar secondary metabolite. Thus this may have been responsible for the relative similarities in the activities of the two species of Streptomyces that were sequenced.

\section{Streptomyces sparsogenes and} Streptomyces griseoplanus possessed the higher activity among all other streptomyces species tested, and according to literature, each of these organisms is known to produce highly potent broad spectrum antibiotic substances such as sparsomycine and tubercidin by Streptomyces sparsogenes (Zhang et al., 2017), alazopeptin and anticapsin (Boeck \& Shah 1971). Erythromycin which is one of the major secondary metabolite secreted by Streptomyces griseoplanus is on the World health organization list of essential medicines due to its effectiveness and safety in health care delivery. Its major mechanism of action is its interference with production of functional proteins which form the basis of its antimicrobial action (Dinos, 2017).

GC-MS analysis of metabolite from Streptomyces sparsogenes confirmed and identified the presence of 10 compounds as a result of the fragmentation pattern observed which in turn was used to confirm their chemical structures. The high antibacterial activity of the most active metabolite (A10) which outweighed even conventional antibiotics may be attributed to the presence of fumaric acid as one of the compounds present in it. From literature, fumaric acid is used primarily in liquid pharmaceutical preparations as an acidulant and flavoring agent. It has also been established for use as a chelating agent which exhibits synergism when used in combination with other true antioxidants (Das et al., 2016). Fumaric acid has been investigated as bioadhesive microspheres due to its hydrophobic nature, which is a necessary property that aids adherence of the agent to microbial surfaces and thus permeating microbial cell wall and membrane (Tango et al., 2015). It has also been used in film-coated pellet formulations as an acidifying agent and also to increase drug solubility. Furthermore, the presence of several organosilicone compounds present in the metabolite may have added to the antibacterial activity recorded. From the GCMS analysis it was evident that seven organosilicone compounds were present at varying peaks of the spectra. According to Mary and Giri (2017) organosilicone compounds possess antimicrobial and antiseptic properties.

This study has established that Streptomyces isolated from dumpsite soils within Ilorin metropolis possessed the potential to produce antibacterial metabolites with activity against pathogenic bacteria. It has also demonstrated that the fermentation carried out on isolates produced bioactive metabolites with enhanced antibacterial activity. The different species of Streptomyces exhibited varying antibacterial abilities indicating that they may have produced different antibacterial compounds 
with varying mechanisms and modes of action responsible for their respective activities.

\section{CONCLUSION}

This study has demonstrated that metabolite produced from S. sparsogenes possessed highest antibacterial activity which inhibited clinical pathogens better than conventional antibiotics suggesting it as a viable source of antibiotics which can be harnessed in treatment of microbial infections. Furthermore, the GC-MS analysis revealed the presence of active compounds such as fumaric acid which is a chelating agent that can be harnessed for pharmaceuticals.

\section{ACKNOWLEDGEMENT}

Authors wish to acknowledge the support of the University of Ilorin through research funding scheme UIL/FLS/MCB/18/082.

\section{REFERENCES}

Abbas KH., Al-Snaf AE. \&Bander KI. (2009). Isolation and Identification of Antibiotics Produced by Penicillium brasilium Batista Isolated from Salahaddin Province Soils. Thi-Qar Medical Journal (TQMJ) 3: 71-87.

Abdulkadir M. \& Waliyu S. (2012). Screening and isolation of the soil bacteria for ability to produce antibiotics. European Journal of Applied Science 4(5): 211-215.

Adegboye MF. \& Babalola OO. (2013). Isolation, characterization and antibacterial activity of Streptomycetes from rhizosphere soils in North West Province, South Africa. The Asian International Journal of Life Sciences 9: 403-421.

Ahmed RN., Abdullahi MA., Zakariyah RF., Gambari-Ambali RO., Adeyemi SB., Afonja AI. \& Adebisi OO. (2015). Antibacterial Action of Silver Nano Particles of Extract of Thevetia nerifolia. International Journal of Phytofuels and Allied Sciences4(1): 31-55.

Ajijolakewu K., Leh C., Wan AbduLLah W. \& Lee C. (2016). Assessment of the Effect of Easily-metabolised Carbon Supplements on Xylanase Production by Newly Isolated Trichoderma asperellum USM SD4 Cultivated on Oil Palm Empty Fruit Bunches. BioResources11(4): $\quad$ 9611-9627. doi:10.15376/biores.11.4.9611-9627

Aliero AA., Emmanuel E., Josephat MN., Sambo HA., Matilda AO. \& John O. (2017). Antibacterial Activity of Actinomycetes Isolated from Waste Dump Soil from Western Uganda. Microbiology Research Journal International 21(5): 1-14.

Ashok KP. \& Karpagam P. (2016). Screening of Antibiotic Producing Actinomycetes from the Sediments of Undisturbed Forest Areas and Its Hyper Activity after Mutation. International Journal of Advances in Chemical Engineering and Biological Sciences 3(2): 46-43.

Bizuye A., Moges F. \& Andualem B. (2013). Isolation and screening of antibiotic producing actinomycetes from soils in Gondar town, North West Ethiopia. 
Asian Pacific Journal of Tropical Diseases 3(5): 375-381.

Bizuye A., Bii C., Erastus G. \& Maina N. (2017). Antibacterial metabolite prospecting from actinomycetes isolated from damped soils from Thika central part of kenya. Asian Pacific Journal of Tropical Diseases 7(2): 757-764.

Boeck LD., Christy KL. \& Shah R. (1971). Production of anticapsin by Streptomyces griseoplanus. Applied microbiology 21(6): 1075-1079.

Chaudhary HS., Yadav J., Shrivastava AR., Singh S., Singh AK. \& Gopalan N. (2018). Antibacterial activity of actinomycetes isolated from different soil samples of Sheopur (A city of central India). Journal of Advanced Pharmaceutical Technology and Research 4(2): 118-123.

Das RK., Brar SK. \& Verma M. (2016). Fumaric Acid: Production and Application Aspects. Platform Chemical Biorefinery 8: 133-157.

Dinos PG. (2017). The Macrolide Antibiotics Resistsnce. British Journal of Pharmacology 174: 2967-2983.

Emami E., Issazadeh K., Akhavan SA. \& Tajehmiri A. (2017). Isolation and Characterization of Aerobic Actinomycetes from Soil in Northern Iran and Evaluation of their Antimicrobial Potential J Med Bacteriol 6(3 \&4): 14-22.
Fawole MO. \& Oso BA. (2007). Laboratory manual of microbiology, $5^{\text {th }}$ edition spectrum book limited, Ibadan, Nigeria, pp. 16-33.

Fay GD. \& Barry AL. (1974). Methods for detecting indole production by gramnegativenon-spore forming anaerobes. Applied Microbiology 27(3): 562-565.

Kaur S., Kaur HP. \& Kaur G. (2016). Isolation and characterization of antibiotic producing actinomycetes from agriculture soil. World Journal of Pharmacy and Pharmaceutical Sciences 5(6):1109-1117.

Njenga WP., Mwaura FB., Wagacha JM. \& Gathuru EM. (2017). Methods of isolating actinomycetes from the soils of Menengai crater in Kenya. Archives of Clinical Microbiology 8: 3.

Manganyi MC., Tchatchouang CK., Regnier T., Bezuidenhout CC. \& Ateba CN. (2019) Bioactive Compound Produced by Endophytic Fungi Isolated from Pelargonium sidoides Against Selected Bacteria of Clinical Importance. Mycobiology 47(3): 335339.

Mary PA. \& Giri RS. (2017). GC-MS Analysis of Bioactive Compounds of Acryranthes aspera. World Journal of Pharmaceutical Research 7(1): 10151056.

Ohnishi Y., Ishikawa J., Hara H., Suzuki H., Ikenoya M., Ikeda H., Yamashit A., 
Hattori M. \& Horinouchi S. (2008). Genome sequence of the streptomycin- producing microorganism Streptomyces griseus IFO 13350. Journal of Bacteriology 190(11): 4050-4060.

Raja A. \& Prabakarana P. (2011). Actinomycetes and Drug-An Overview. American Journal of Drug Discovery and Development 1: 75-84.

Sathi ZS., Rahman MAA. \& Gafur MA. (2001). Identification and in vitro antimicrobial activity of compound isolated from Streptomyces species. Pakistanian Journal of Biological Science 4: 1523-1525

Sharma P., Pajni S., Dhillon N., Vadehra D. \& Dube D. (1986). Limitations of the Congo-Red staining techniques for the detection of cellulolytic activities. Biotechnology letters 8(8): 579-580.

Sharma M., Dangi P. \& Choudhary M. (2014). Actinomycetes: Source, Identification, and Their Applications. International Journal of Current Microbiology and Applied Sciences 3(2): 801-832.

Sikander A., Maria N., Aiman TL., Maryam S. \& Attia M. (2018). Production of Microbial Metabolites and Optimization of Key Factors Involving their Hyperproduction in
Batch Culture. European Journal of Pharmaceutical and Medical Research 5(5): 80-88.

Simon C. \& Daniel R. (2011). Metagenomic analyses: past and future trends. Applied and Environmental Microbiology 77(4): 1153-1161.

Singh AP., Singh RB. \&Mishra S. (2012). Studies on isolation and characterization of antibiotic producing microorganism from industrial waste soil sample. The Open Nutraceutical Journal 5: 169172.

Tango CN., Mansur RA. \& Oh DH. (2015). Fumaric Acid and Slightly Acidic Electrolyzed Water Inactivate Gram Positive and Gram Negative Foodborne Pathogens. Microorganisms 3(1): 34-46

Zhang Hongyu., Qiuling Zhou., Tingting Lou., Suying Wang. \& Haihua Ruan. (2017). Draft genome sequence of broad-spectrum antibiotic sparsomycin-producing Streptomyces sparsogenes ATCC 25498 from the American Type Culture Collection. Journal of Global Antimicrobial Resistance 11: 159-160.

Ziemert N. \& Jensen PR. (2012). Phylogenetic approaches to natural product structure prediction. Methods in enzymology517, 161-182. 